\title{
Preliminary Design of the Support Structure for a Rotating Carbon-Ion Transfer Line for Medical Applications
}

\author{
Diego Perini ${ }^{1}$, Luca Dassa ${ }^{1}$, Luca Piacentini ${ }^{2, *}$ and Stefano Uberti ${ }^{3}$ \\ 1 CERN, 1211 Geneva, Switzerland; diego.perini@cern.ch (D.P.); luca.dassa@cern.ch (L.D.) \\ 2 Center of High-Energy Physics and Accelerator Technologies, Riga Technical University, LV-1048 Riga, Latvia \\ 3 Department of Mechanical and Industrial Engineering, University of Brescia, 25123 Brescia, Italy; \\ stefano.uberti@unibs.it \\ * Correspondence: luca.piacentini@rtu.lv
}

Citation: Perini, D.; Dassa, L.;

Piacentini, L.; Uberti, S. Preliminary Design of the Support Structure for a Rotating Carbon-Ion Transfer Line for Medical Applications. Instruments 2021, 5, 34. https://doi.org/10.3390/ instruments5040034

Academic Editor: Antonio Ereditato

Received: 20 October 2021

Accepted: 24 November 2021

Published: 27 November 2021

Publisher's Note: MDPI stays neutral with regard to jurisdictional claims in published maps and institutional affiliations.

Copyright: (c) 2021 by the authors. Licensee MDPI, Basel, Switzerland. This article is an open access article distributed under the terms and conditions of the Creative Commons Attribution (CC BY) license (https:/ / creativecommons.org/licenses/by/ $4.0 /)$.

\begin{abstract}
The development of new bent superconducting magnets together with the optimization of the support structure open the way to a considerable reduction in the weight and complexity of rotating gantries for medical applications. The magnets, which define the transfer line to deliver carbon ions to the patients from different angles, are supported by a rotating structure that should be as rigid and as lightweight as possible. Relative displacements of the magnets due to deformations cause incorrect beam position and consequent errors in hitting the target tissues. This paper describes a possible rotating structure which is considerably lighter than the previous designs. A method to compensate part of the deformation by complementary rotations of the driving motor is proposed. The influence of the construction tolerances and deformations of the supports is also analyzed and alignment and adjustment possibilities are discussed.
\end{abstract}

Keywords: gantry for medical applications; ion therapy; curved magnets

\section{Introduction}

Carbon ion therapy offers new possibilities for the medical treatments of some cancers [1]. This is due to the specific profile of energy loss of these ions as they travel through human tissues, the so-called Bragg peak. Carbon ions, compared with other treatment beams, present a very pronounced peak. In other words, most of the beam energy is delivered at a given depth in the tissues and only minor doses are dissipated in the tissues before and after the cancer target. The depth of the peak can be tuned according to the energy of the incoming carbon beam. In this way, the cancer cells are killed by the beam, whilst minimizing the damage to the other tissues in the patient's body.

The carbon beam is produced and stored in a compact synchrotron [2] and then extracted and delivered to the patient via a transfer line called a "gantry". The gantry must rotate around the patient to hit the target tissues from different angles. Currently, there are only a few facilities in the world able to rotate and deliver carbon beams [3]. These gantries are large structures, with counterweights to have a perfectly balanced axisymmetric element. The total weight is on the order of $600 \mathrm{t}$, in the case of the HIT, Heidelberg structure [4], and $300 \mathrm{t}$ for HIMAC, Chiba, which is a more recent facility [5], thanks to the first implementation of superconducting magnets.

A simplified gantry, with no counterweight and using curved superconducting magnets, has been proposed [2]. In this paper, we present the first studies concerning the rotating gantry support structure.

In general, in accelerators and transfer lines, charged particles travel in vacuum and are kept in a given trajectory by the magnetic field generated by a set of magnets. The magnets must be properly aligned to drive the particles in the correct direction. A misalignment of the magnets in a gantry by a few millimeters could lead to the beam missing the target and hitting other tissues than the tumor. Small misalignments can be 
corrected by the correctors, other compensation magnets present on the transfer line, but the correction range is limited to fractions of millimeters. The tolerances on the magnets' displacements were assumed to be coherent with the one required by designs of previous gantries [4]. During the rotation of a gantry, the support structure deforms under its own weight and the weight of the magnets. These loads along with the couple generated by the rotating motor and the related brakes, deform the supports and the wall supporting the system. Fabrication and assembly tolerances also influence the final shape and position of the rotating structure.

All these displacements add to the theoretical rotation and define the real position of the magnets and consequently of the carbon ion beam. For this reason, it is important to minimize the deformations of the wall, supports and rotating structure system that cause magnet misalignments. We demonstrate below that the total deformation can be divided into two parts: an extra rotation of the gantry, which is easy to compensate, and a twist of the gantry that cannot be compensated and must be as low as possible.

\section{Method of Analysis}

\subsection{Deformations}

As already said, the goal is to minimize the relative deformation between the magnets of the transfer line (the transfer line was schematized by the following masses: $500 \mathrm{~kg}$ for quadrupoles and scanning magnets, $1000 \mathrm{~kg}$ for the three SC dipoles and $200 \mathrm{~kg}$ for the nozzle; note that the principles contained in this paper remain generally valid regardless of the chosen absolute values). This is achieved by introducing the compensation of part of the deformations through a complementary rotation $\delta_{\text {rigid }}$ of the drive motor.

This process is explained in Figure 1. The dotted and continuous black lines on the left side represent the supports connecting the gantry to the wall. The green dotted line represents the undeformed gantry (as if it was infinitely rigid) in the position $\theta$. The curved black line containing the point $P^{\prime \prime}$ is the gantry in the final position including its own deformations and those of the supports. The point $P$ represents the fixation point of one of the magnets in the line. Due to the deformations, it would end in the position $P^{\prime \prime}$ if nothing is done. The point $P^{\prime \prime}$ could be brought back to its nominal position $P$ by a rotation $\delta_{\text {rigid }}$ and a translation $\eta_{\text {rigid }}$.

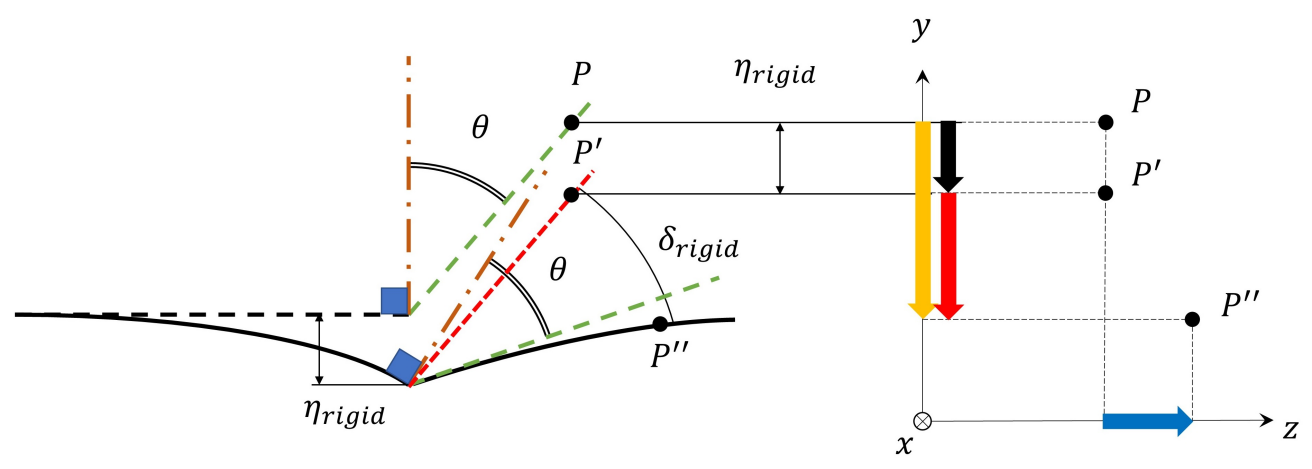

Figure 1. Scheme of the deformation of the assembly (structure + main supports). Here, $P$ is the theoretical position of a given point in the gantry. $P^{\prime \prime}$ is the real position due to the deformations of the structure and supports.

The scheme in Figure 1 assumes an infinitely rigid wall. This is similar to the situation proposed in Figure 2, with the motor on its own support in a dedicated wall and a double cardan joint to connect it to the gantry. The deformation of the main supports is independent from the angular position of the structure $\theta$. Therefore, the supports contribute to the overall deformation only by a rigid translation $\eta_{\text {rigid }}$ (black arrow). This displacement can be compensated by an opposite rigid translation of the main supports during their assembly. This procedure is necessary only in the beginning since $\eta_{\text {rigid }}$ is a function of the weight only. 


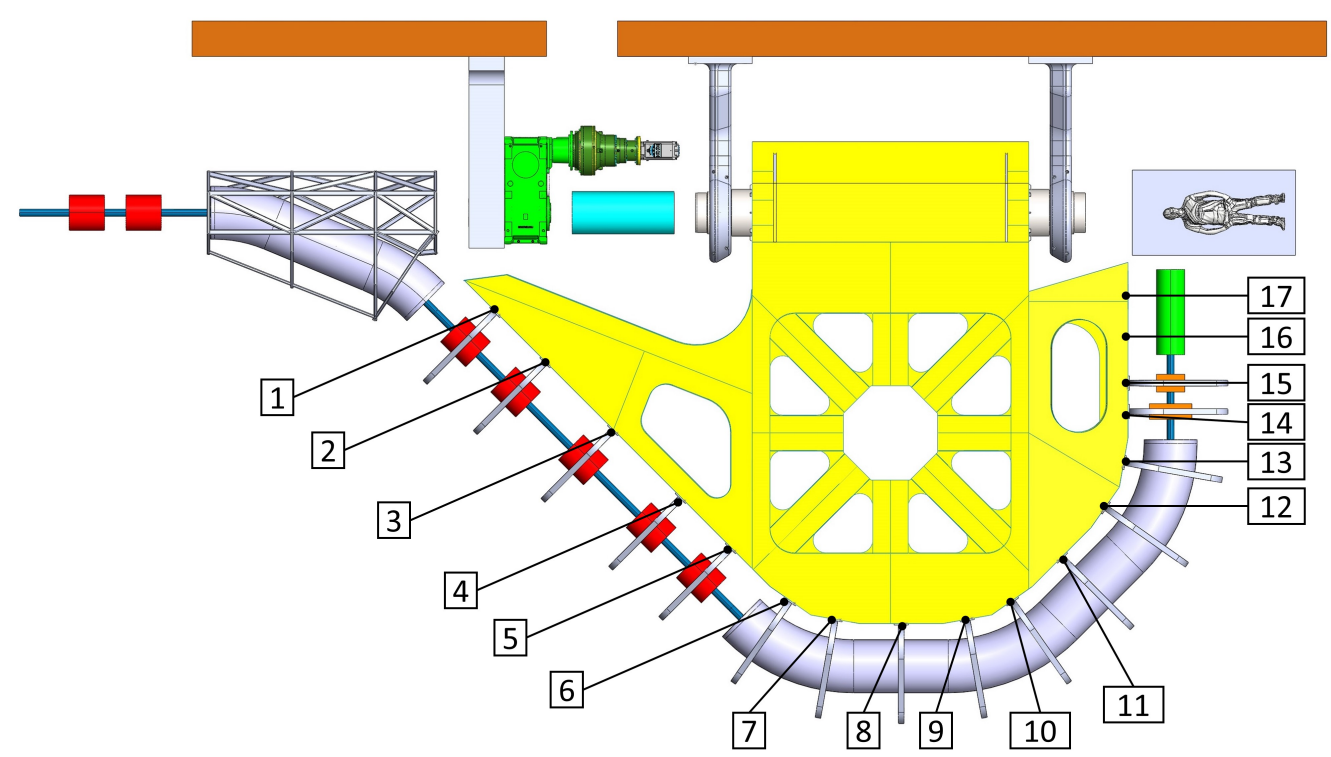

Figure 2. Top view of the gantry structure (yellow) in the horizontal position $\theta=90^{\circ}$. The structure is hinged to two supports fixed to the wall and connected to the motor by a double cardan joint (cyan). The drive motor is fixed on a third support on a dedicated wall. The numbers enumerate nodes of which displacements will be analyzed.

Figure 1 refers to a plane perpendicular to the gantry rotation axis; $\eta_{\text {rigid }}$ is constant for any plane perpendicular to the rotation axis, $\delta_{\text {rigid }}$ can vary from plane to plane, due to the twist of the gantry. In the following paragraph we explain how an average $\delta_{\text {rigid }}$ is calculated to minimize magnets' misalignments.

After a statical Finite Element Analysis (FEA) of the assembly, carried out with the software SOLIDWORKS ${ }^{\mathrm{TM}}$, the displacements of all nodes (in the fixed reference system) are imported in a spreadsheet. Then, $\eta_{\text {rigid }}$ is subtracted from the $u_{y}$ component (where $u_{y}$ is the vertical component of the displacement in the fixed reference system (Figure 3)), and the displacements are converted in the rotating reference system (Figure 3). Finally, the out-ofplane component $u_{t}$ (the tangential component of the displacement in the rotating reference system (Figure 3)) of only those nodes close to the magnets' supports (the supports of the magnets and cryostats have not been studied in this paper; the FEA analysis takes into account their loads as masses rigidly connected to some selected nodes of the structure) is plotted as function of the radial position (see Figure 4). The interpolation of these points by a straight line from the origin gives the average angle of rigid rotation $\delta_{\text {rigid }}$ that will be compensated by the motor.

The average out-of-plane displacement of nodes that share the same $x$ and $r$ coordinates (called Nodes Average Displacement or NAD) is then plotted along the axial coordinate, together with the rigid component that is found by multiplying $\delta_{\text {rigid }}$ and the radial position of that same node (Figure 5). Since the rigid component can be compensated by the drive motor, the only relative deformation between the node deformed position and the theoretical one is obtained by subtracting the rigid displacement from the NAD, and is therefore called "non-rigid" displacement. This procedure is iterated to optimize the structure by minimizing non-rigid displacements. It is important to underline that the non-rigid component is an order of magnitude smaller than the NAD. This is because of the innovative solution proposed for compensating rigid rotations. 


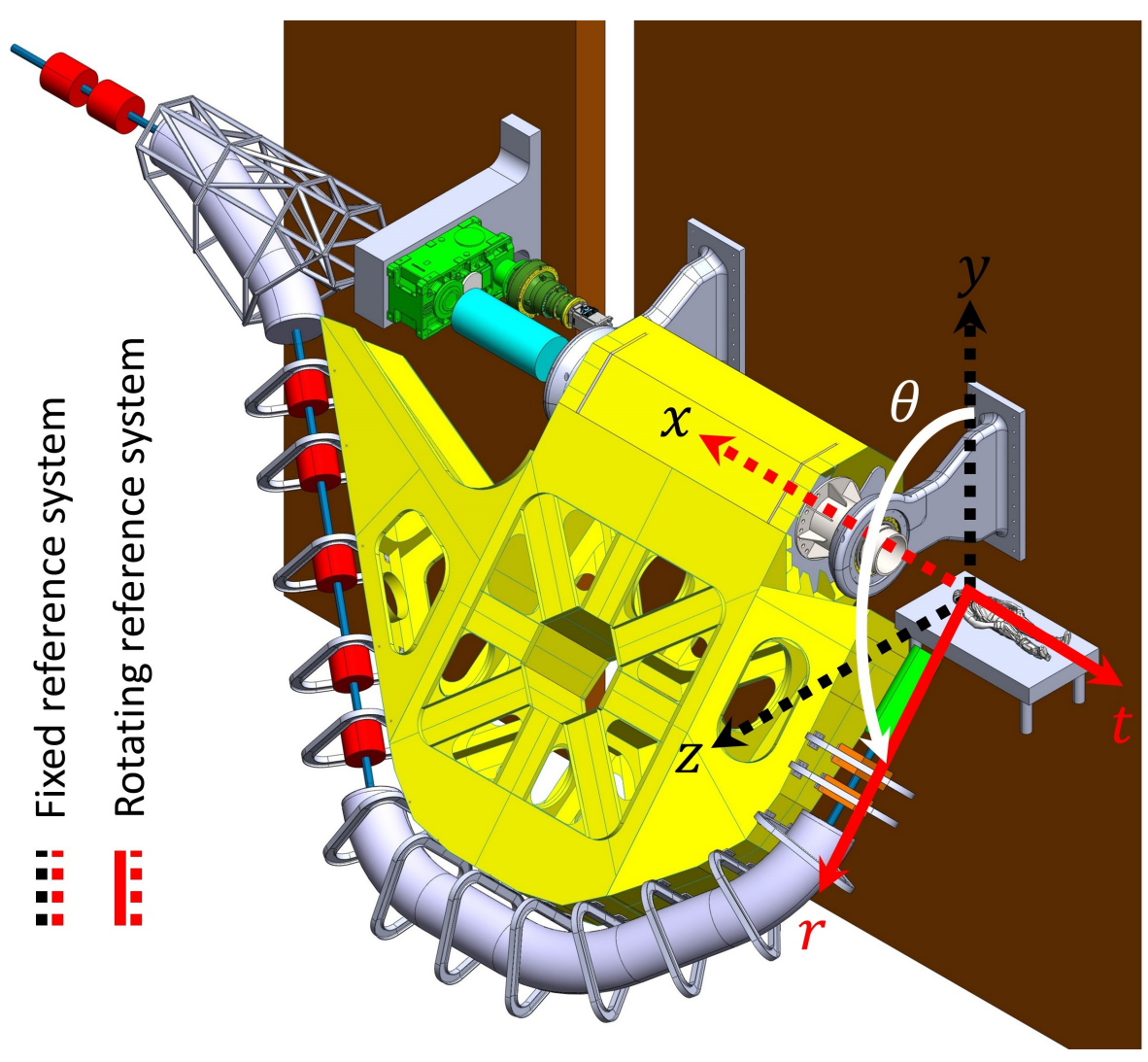

Figure 3. Cartesian reference systems used in the study of the deformations of the structure and its main supports: a fixed system of reference, dotted, and a rotating system of reference highlighted in red. The structure is rotated by $\theta=135^{\circ}$, where $\theta$ is the angle measured from $y$ to $r$.

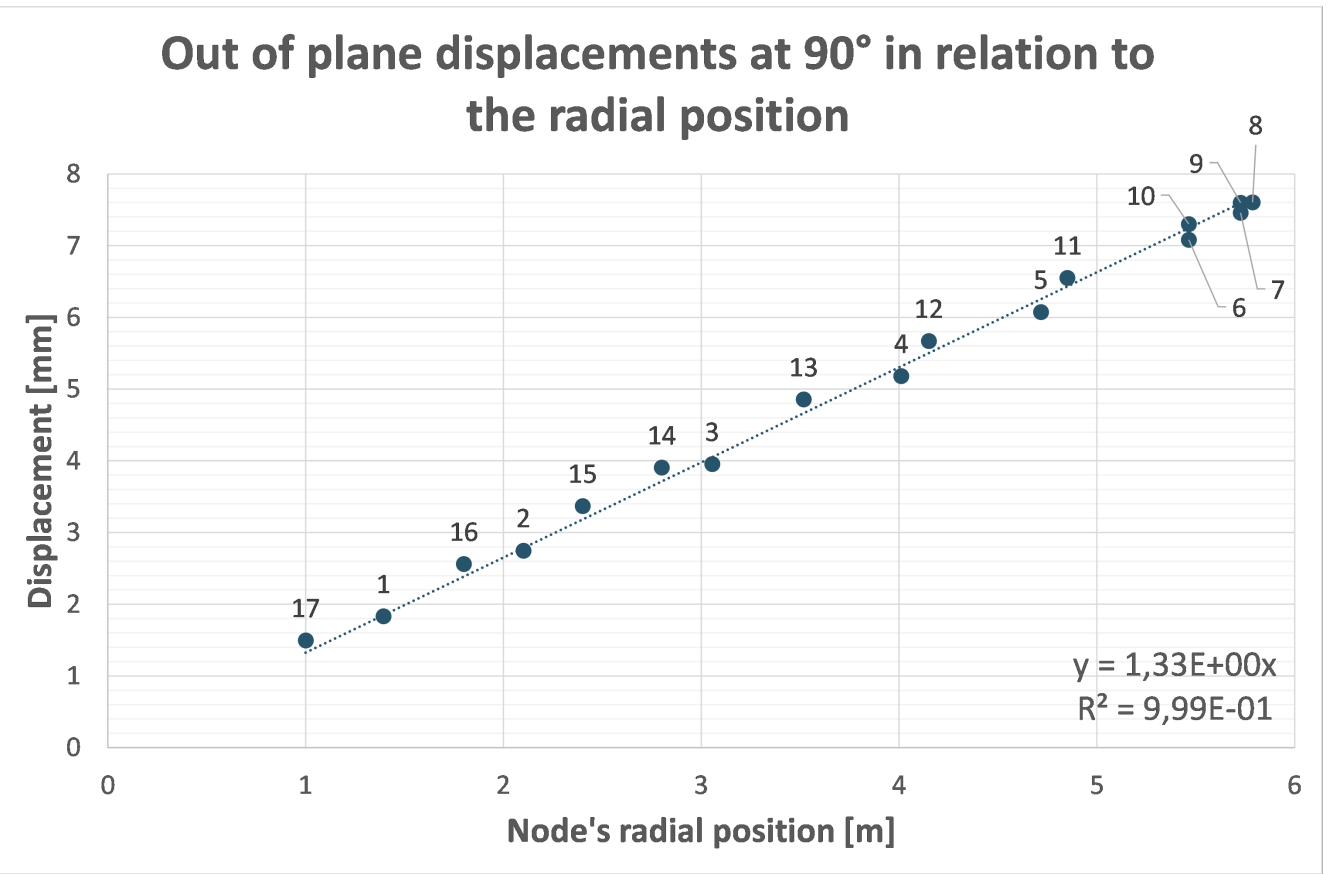

Figure 4. Out-of-plane displacements of some selected nodes along the magnet line when the structure is in a horizontal position $\left(90^{\circ}\right)$. 


\section{Out of plane displacements at $90^{\circ}$ along the axis of} the structure

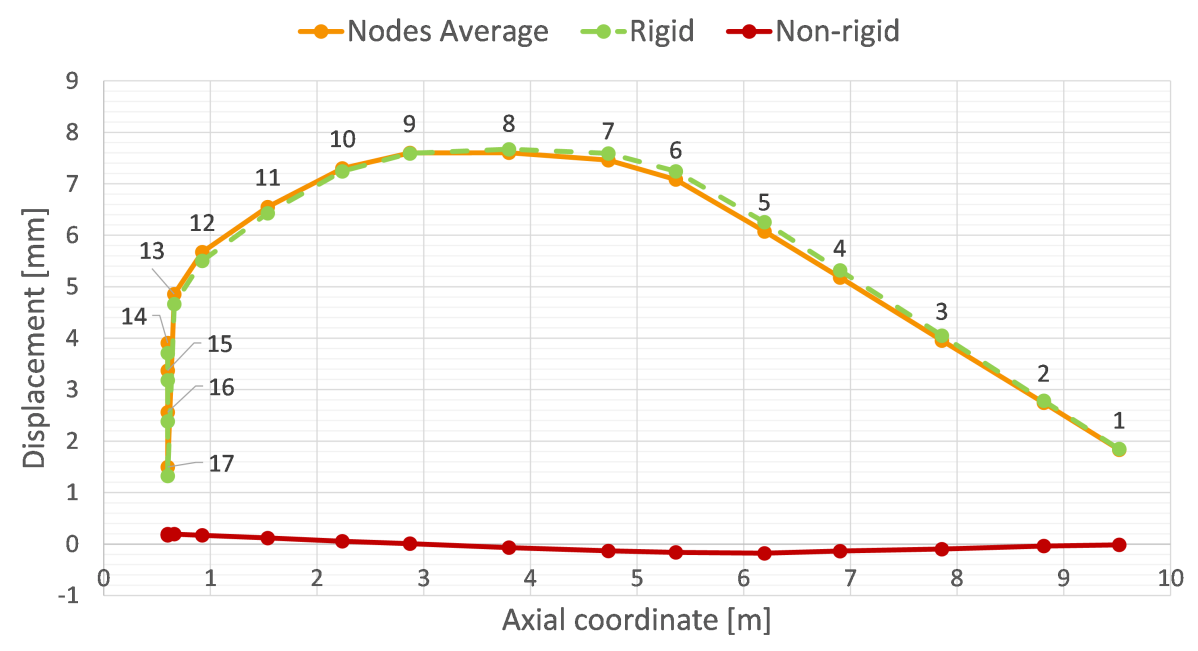

Figure 5. Components of the out-of-plane displacement when the structure is at $90^{\circ}$ : NAD in (orange), theoretical rigid displacement (dashed green) derived from the interpolation of NAD with respect to the $\mathrm{r}$ coordinate, non-rigid displacement obtained by subtracting the rigid component from NAD (dark red).

\subsection{Tolerances}

Another aspect that influences the real position of the magnets with respect to the theoretical one is the chain of tolerances of the different components. The total tolerance range is computed in two ways: by hand and by software computation (using the "Tolanalyst" tool provided by the SOLIDWORKS ${ }^{\mathrm{TM}}$ package). In both cases, the error is calculated using the "worst case" and statistical approach, in the first method, all dimensions are assumed at the extreme limit. Therefore, the resulting maximum or minimum error can be calculated as an arithmetic sum (or subtraction). In the second method each dimension is supposed to have a bell or normal distribution.

The tolerance chain is the result of the contribution from the wall, the main supports, the bearings and the structure. The problem can be divided into two parts thanks to the application of the superposition principle. The rotation axis is the border between the two sub-chains. We have one sub-chain from the wall to the axis (upstream) and the other from the axis to the support structure (downstream) (Figure 6).

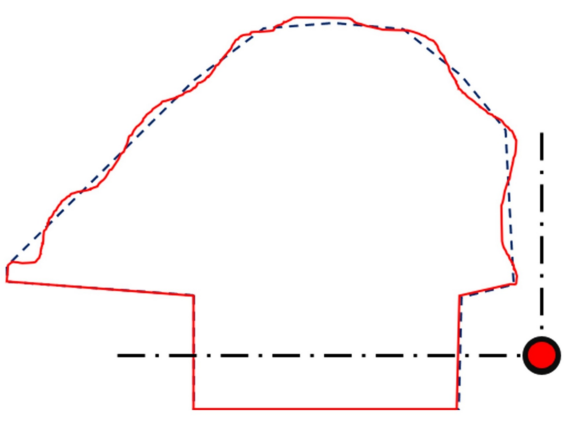

(a)

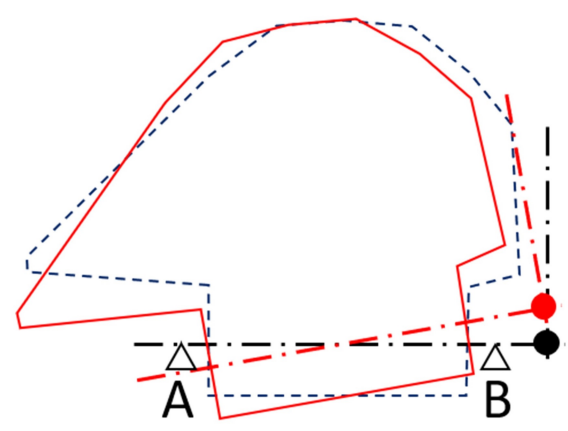

(b)

Figure 6. (a) Effect of tolerances chain considering only those from the downstream axis of the structure. (b) Effect of tolerances chain considering only those from the upstream axis, passing through the bearings' centers. 
The first sub-chain is related to the deviation of the structure from its theoretical shape, while still rotating around the nominal axis. The second one is related to the deviation of the rotation axis from its nominal position, while the structure is considered perfect. The superposition of the two gives the total.

The two contributions can be partially or totally compensated by tuning systems. The downstream tolerances can be corrected by adjustable supports in between the structure and the magnets. The upstream contributions can be corrected by shims and rails on the support wall. This paper analyzes only the second contribution to estimate the order of magnitude of the error. This will provide the input for the design of the compensation mechanisms.

For the second group of tolerances, we consider the two components giving the maximum vertical and horizontal deviation, respectively measured along the $y$ and $z$ axes. Errors along the $x$ direction have a smaller effect on the position of the axis of rotation, and are therefore ignored.

The greatest contribution to the maximum vertical deviation $\eta_{y}$ is given by the relative rigid rotation of the main supports (Figure 7) due to the lever arm. In particular, the worst case occurs when the two are counter-rotated, as illustrated in Figure 7. In that case, the wall behind each support is inclined and reaches the upper and lower limits of the planar tolerance, as illustrated in Figure 7.

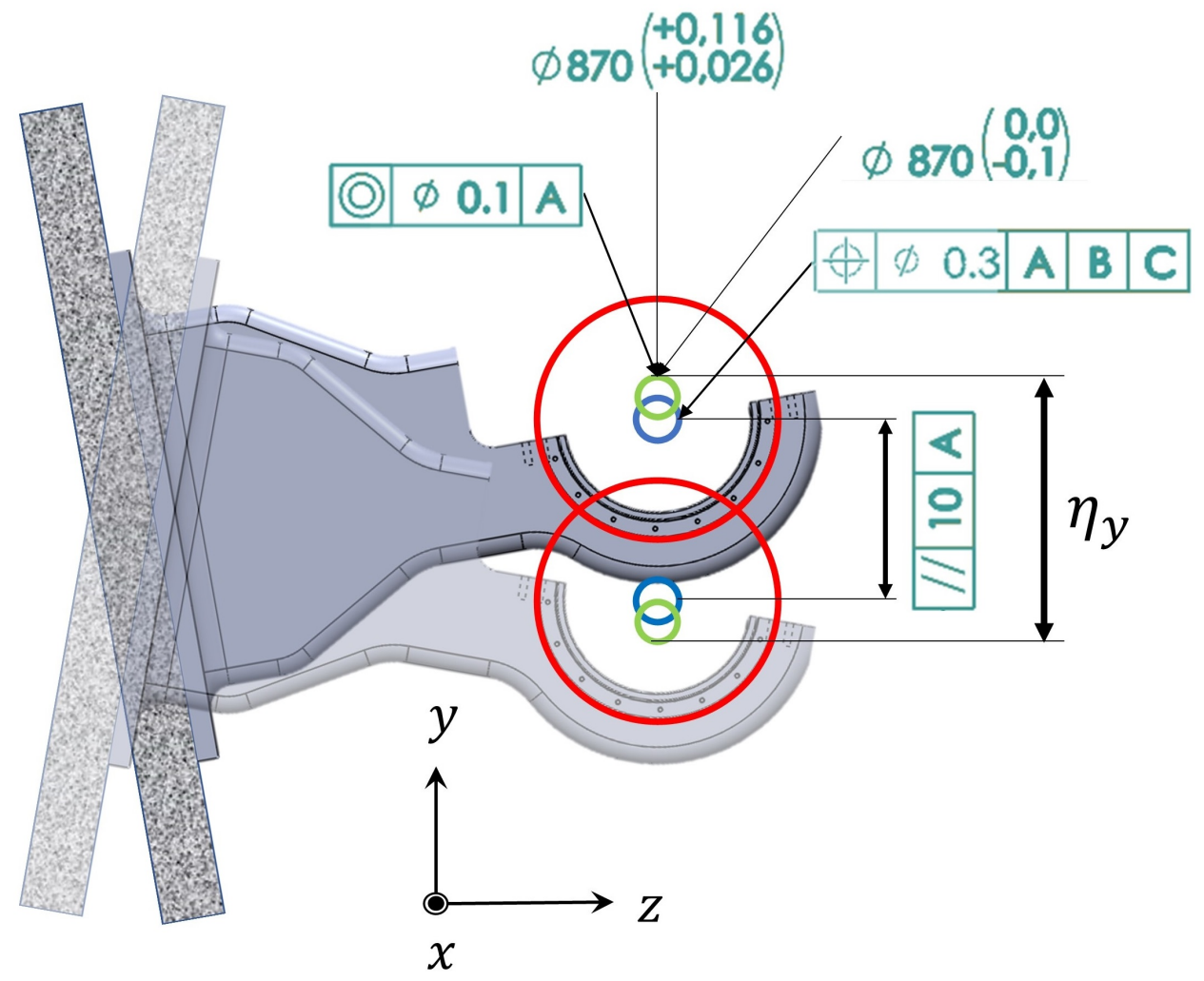

Figure 7. Vertical deviations: scheme of the effect of tolerances on the position of the centers of the bearings.

The maximum horizontal error $\eta_{z}$ occurs when the wall behind each support is at the upper and lower boundary condition of the planar tolerance and the two contact regions are still parallel (see Figure 8).

The calculation along both directions are given in Appendix A. 


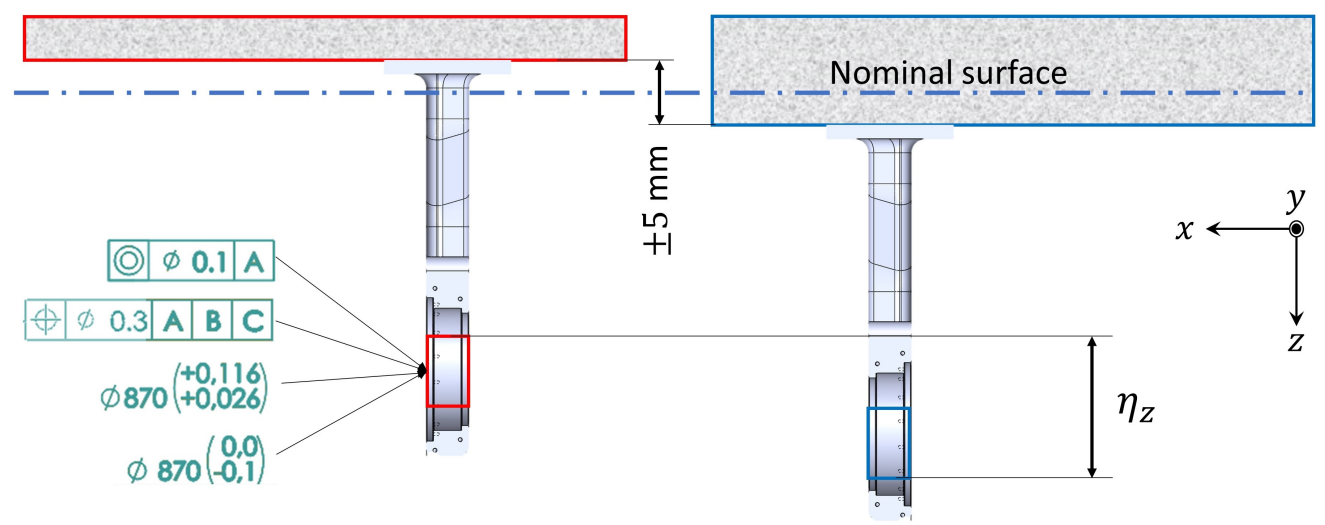

Figure 8. Horizontal deviations: scheme of the effect of tolerances on the position of the centers of the bearings. The blue and red rectangles in the bearings represent a qualitative illustration of the region where it is possible to have the center of each bearing.

\section{Results}

\subsection{Deformations}

The structure supporting the magnet line has been optimized to minimize its weight and the deformations during the rotation [6]. The most critical position, in terms of deformations, is with the system in the horizontal position $\left(\theta=90^{\circ}\right)$. This is also the configuration in which there are maximal torque loads on the support wall. The deformations of the supports and wall cause deviations of the magnet position with respect to the theoretical position and add to the deformations and tolerances of the gantry itself. To avoid as much as possible the wall and support contribution, it has been proposed to structurally disconnect the reinforced concrete supporting structure of the motor from that of the gantry rotating frame. The motor and the rotating structure are connected by a double cardan joint (Figure 2).

In this way, we can separate the functions. The torque generated by the cantilever position of the gantry is transmitted by the cardan joints and the motor to the wall separated from the supports. This wall deforms but does not influence the deformation of the supports that are loaded by the gantry weight only. The load on the supports is then independent from the angular position of the gantry.

In Figure 9, the displacements along the axial, radial and "out-of-plane" directions are given for different rotation angles of the gantry. After compensation of the rigid rotation, the only component of the out-of-plane displacement giving misalignment of the magnets is the non-rigid one. The non-rigid displacement is of a lesser order of magnitude than the average displacement (Figure 5).

\subsection{Tolerances}

The results of the study of tolerances, explained in Section 2.2 and calculated by hand in Appendix A, are shown in Table 1.

The rotation of the axis (in the $x-y$ plane) given by the maximum vertical deviation can produce an error for the center position of up to $\simeq 21 \mathrm{~mm}$. The contribution of the wall precision is quite important ( $\simeq 97 \%$ using the worst case approach). The assumed precision of the wall can seem pessimistic but in any case, for civil engineering, realistic tolerances are at least one order of magnitude larger than what we can expect for mechanical components. The problem can be solved by designing a bi-directional, adjustable system for the supports. This solution also helps compensate the deformation of the supports. These errors need to be compensated only once during the assembly phase, both in the case of deformations and tolerance deviations. 


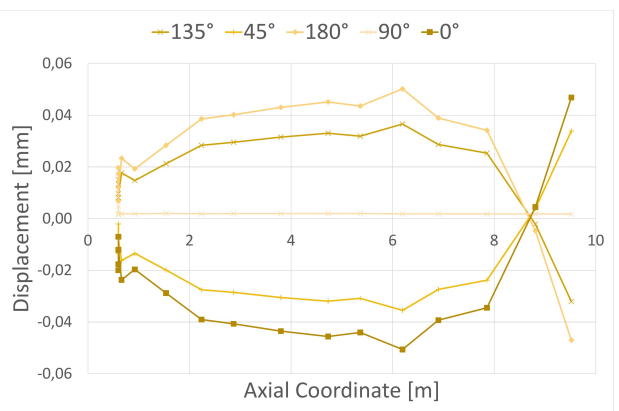

(a)

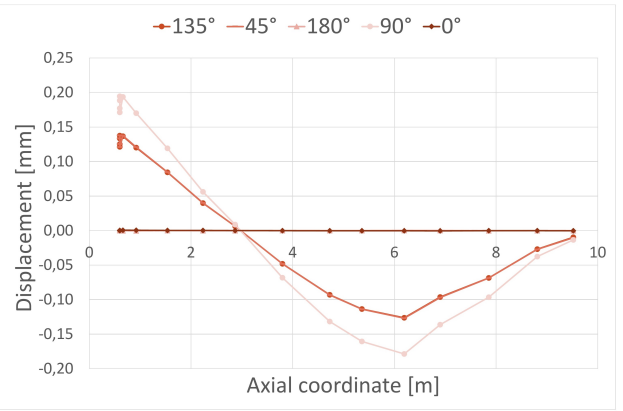

(c)

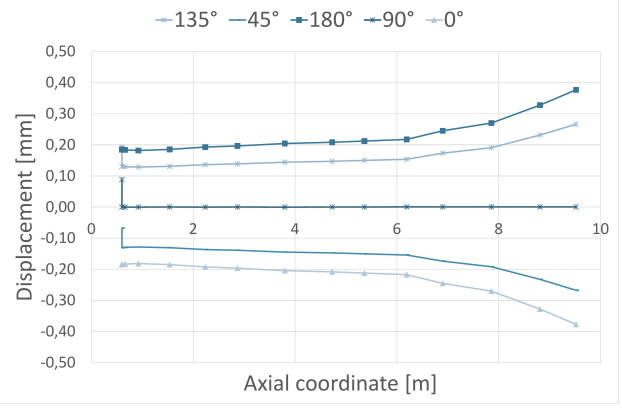

(b)

Figure 9. Comparison of the displacements of relevant nodes between different angular positions of the gantry, where each component is measured in the rotating reference system along the $x$ axis: (a) axial displacement. (b) Radial displacement. (c) Out-of-plane non-rigid displacement. See Figure 5 for nodes-average and rigid displacements.

Table 1. Results of the evaluation of vertical and horizontal deviations between the main supports due to the stack-up problem. The results are shown for both the handmade model and the software analysis.

\begin{tabular}{lccc}
\hline Handmade Model & “Worst Case" & Statistical & \\
\hline Maximum vertical deviation & 20.62 & 14.14 & $\mathrm{~mm}$ \\
Maximum horizontal deviation & 10.62 & 7.07 & $\mathrm{~mm}$ \\
\hline Software Analysis & “Worst Case" & Statistical & \\
\hline Maximum vertical deviation & 20.73 & 14.14 & $\mathrm{~mm}$ \\
Maximum horizontal deviation & 10.63 & 7.07 & $\mathrm{~mm}$ \\
\hline
\end{tabular}

\section{Discussion and Conclusions}

Deformations of the wall, the supports and the gantry, and their construction tolerances influence the position of the magnets in the rotating transfer line defining the path of the carbon ions. These perturbations have to be minimized to correctly reach the target in the patient. Several levels of interventions are proposed:

- $\quad$ Decouple the wall portion loaded by the reaction to the torque generated by the cantilever position of the gantry from the wall portion supporting the gantry. The rotating motor is connected to the gantry by a double cardan joint.

- Envisage rails and shims between the wall and the supports to fine-tune their position and compensate their deflection and fabrication tolerances.

- Envisage rails and shims between the gantry and the magnet fixations to compensate for the gantry construction tolerances.

- $\quad$ Consider the gantry deformation as the sum of two components: rotation and twist. The first and larger component can be compensated by a differential rotation of the motor. The second component is one order of magnitude smaller than the first and 
will define the magnet misalignment. This misalignment can be estimated to be of the order of $0.1-0.3 \mathrm{~mm}$. This will cause small deviations from the nominal ion path that are in the range of what can be compensated by additional correction coils.

With these steps, we can reach the necessary final precision compensating the nonideal behavior by tuning elements and without imposing severe tolerances that would imply high fabrication costs.

Author Contributions: Conceptualization, D.P., L.D. and S.U.; Data curation, L.P.; Formal analysis, L.P.; Methodology, D.P., L.D., L.P. and S.U.; Software, L.P.; Supervision, D.P., L.D. and S.U.; Writingoriginal draft, D.P. and L.P.; Writing - review \& editing, D.P., L.D. and S.U. All authors have read and agreed to the published version of the manuscript.

Funding: This research received no external funding.

Institutional Review Board Statement: Not applicable.

Informed Consent Statement: Not applicable.

Data Availability Statement: Not applicable.

Acknowledgments: The authors wish to thank Maurizio Vretenar for the support he provided for this work. A big thank you to the members of the TERA foundation, CNAO foundation and Riga Technical University (in particular, Elena Benedetto, Ugo Amaldi, Pierluigi Riboni, Marco Pullia, Enrico Felcini and Toms Torims) for the interesting and useful discussions, inputs and suggestions.

Conflicts of Interest: The authors declare no conflict of interest.

\section{Appendix A}

In both cases discussed in Section 2.2, the contributing tolerances are:

- The profile tolerance of a surface on the wall, that is assumed to have a value of $10 \mathrm{~mm}$;

- The position of the bearing housing hole (assumed within $0.3 \mathrm{~mm}$ range, Figure A1);

- The dimensional tolerance on the diameter of the bearing housing hole (of $870_{+0.026^{\prime}}^{+0.116}$ Figure A1);

- The dimensional tolerance on the external diameter of the bearing (of $870_{-0.1}^{+0}$, Figure A2);

- The concentricity on the internal diameter of the bearing (within a range of $0.1 \mathrm{~mm}$, Figure A2).

The tolerance on the wall, however, cannot be evaluated by the software, as it requires a tolerance of parallelism instead. Since the wall is much higher than the support (which is $2.2 \mathrm{~m}$ ), the parallelism tolerance applied over the total length decreases the range in which the main support could rotate. Therefore, we chose to apply the same value over a maximum length of $2.2 \mathrm{~m}$, to obtain a coherent effect to that of the profile tolerance.

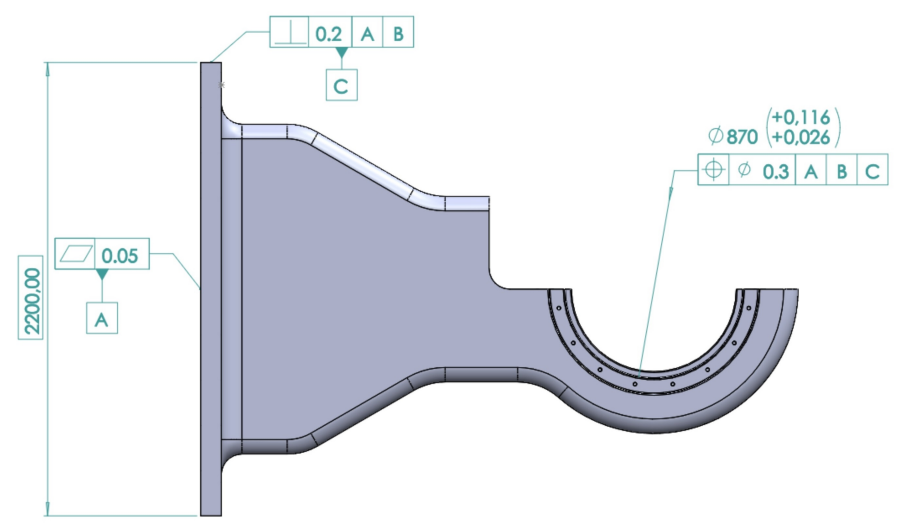

Figure A1. Tolerances of the main support that can influence the tolerance chain problem. 


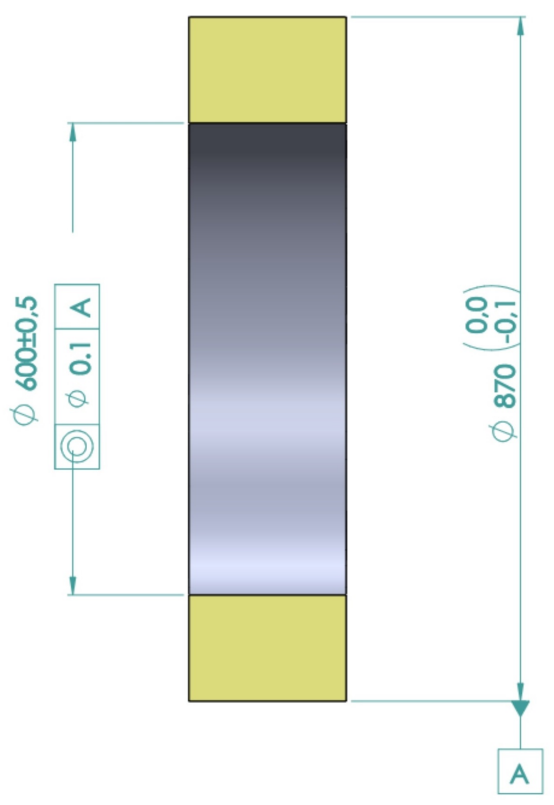

Figure A2. Simplification of the bearing in which the interesting tolerances that can influence the tolerance chain problem are highlighted.

The vertical deviation can be calculated for the "worst case" approach as follows:

$$
\eta_{y}=2\left(t_{\mathrm{par}} \frac{\text { height }}{\text { length }}+\frac{t_{\mathrm{dim}}+t_{\mathrm{con}}+t_{\mathrm{pos}}}{2}\right)=20.62 \mathrm{~mm}
$$

where the ratio "height/length" is the relationship between the height of the support (at the base) and the center distance of the housing hole from the base of the support. Moreover, $t_{\text {par }}, t_{\text {con }}$ and $t_{\text {pos }}$ are the tolerances of parallelism on the wall, the concentricity for the bearing and the position of the bearing's housing, respectively. Finally, $t_{\mathrm{dim}}$ is the maximum clearance between the bearing and its housing hole:

$$
t_{\text {dim }}=D_{\max }-d_{\min }=0.216 \mathrm{~mm}
$$

For the horizontal deviation, the equation is:

$$
\eta_{z}=2\left(\frac{t_{\mathrm{par}}+t_{\mathrm{dim}}+t_{\mathrm{con}}+t_{\mathrm{pos}}}{2}\right)=10.62 \mathrm{~mm}
$$

where a factor of 2 is collected because half the contribution can be considered to be provided by each of the two supports.

On the other hand, a rough statistical approach results in the following deviations:

$$
\begin{aligned}
& \eta_{y, 3 \sigma}=3 \sqrt{2\left[\left(\frac{t_{\mathrm{par}} \frac{\text { height }}{\text { length }}}{3}\right)^{2}+\left(\frac{t_{\mathrm{dim}} / 2}{3}\right)^{2}+\left(\frac{t_{\mathrm{con}} / 2}{3}\right)^{2}+\left(\frac{t_{\mathrm{pos}} / 2}{3}\right)^{2}\right]}=14.14 \mathrm{~mm} \\
& \eta_{z, 3 \sigma}=3 \sqrt{2\left[\left(\frac{t_{\mathrm{par}} / 2}{3}\right)^{2}+\left(\frac{t_{\mathrm{dim}} / 2}{3}\right)^{2}+\left(\frac{t_{\mathrm{con}} / 2}{3}\right)^{2}+\left(\frac{t_{\mathrm{pos}} / 2}{3}\right)^{2}\right]}=7.07 \mathrm{~mm}
\end{aligned}
$$

\section{References}

1. Ohno, T. Particle radiotherapy with carbon ion beams. EPMA J. 2013, 4, 9. [CrossRef] [PubMed]

2. Benedetto, E.; Harbi, N.A.; Brouwer, L.; Tommasini, D.; Prestemon, S.; Riboni, P.; Amaldi, U. A Carbon-Ion Superconducting Gantry and a Synchrotron Based on Canted Cosine Theta Magnets. arXiv 2021, arXiv:2105.04205. 
3. Particle Therapy Facilities in Clinical Operation. Available online: https://www.ptcog.ch/index.php/facilities-in-operation (accessed on 14 January 2021).

4. Fuchs, R.; Weinrich, U.; Sust, E. Assembly of the carbon beam gantry at the Heidelberg Ion Therapy (HIT) accelerator. In Proceedings of the EPAC-2008, Genoa, Italy, 23-27 June 2008.

5. Iwata, Y.; Fujita, T.; Furukawa, T.; Hara, Y.; Matsuba, S.; Mizushima, K.; Murakami, T.; Noda, K.; Saotome, N.; Saraya, Y.; et al. Superconducting Gantry for Carbon-Ion Radiotherapy. In Proceedings of the 9th International Particle Accelerator Conference, Vancouver, BC, Canada, 4 May-29 April 2018; pp. 1232-1236.

6. Piacentini, L. Project Development of a Rotating Transferring Line for Carbon Ions Used for Medical Scope. Master's Thesis, Università degli Studi di Brescia, Brescia, Italy, 2021. 